\title{
Author Correction: Multidimensional nanoscopic chiroptics
}

Yang Chen, Wei Du (D), Oing Zhang, Oscar Ávalos-Ovando (D), Jing Wu (D), Oing-Hua Xu, Na Liu, Hiromi Okamoto (D), Alexander O. Govorov, Qihua Xiong (D) and Cheng-Wei Qiu (D)

Correction to: Nature Reviews Physics https://doi.org/10.1038/s42254-021-00391-6, published online 15 November 2021.

The original version of this article incorrectly showed the affiliation of N.L. This has been corrected to " $2^{\text {nd }}$ Physics Institute, University of Stuttgart, Stuttgart, Germany”. The original article also omitted the acknowledgement of N.L.'s financial support from the Max Planck Society (Max Planck Fellow Program) and Q.X.'s Grant No. 92056204 from the National Natural Science Foundation of China and funding from the State Key Laboratory of Low-Dimensional Quantum Physics. These errors have now been corrected in the HTML and PDF versions of the article.

https://doi.org/10.1038/s42254-021-00405-3 I Published online 22 November 2021

(C) Springer Nature Limited 2021 